\title{
Frequency doubling in periodic nonlinear photonic crystals mediated by random layers
}

\author{
Andrey M. Vyunishev ${ }^{1,2, *}$ AND Anatoly S. Chirkin $3{ }^{3, *}$ \\ ${ }^{1}$ Kirensky Institute of Physics, Federal Research Center KSC SB RAS, Krasnoyarsk, 660036, Russia \\ ${ }^{2}$ Department of Photonics and Laser Technology, Siberian Federal University, Krasnoyarsk, 660079, Russia \\ ${ }^{3}$ Faculty of Physics and International Laser Center, M. V. Lomonosov Moscow State University, Moscow, 119992, Russia \\ *Corresponding author: vyunishev@iph.krasn.ru \\ Compiled January 26, 2017
}

We study the frequency doubling in a quadratic nonlinear photonic crystal consisting of periodically poled structures mediated by random layers. These structures can be formed by new local impact methods for ferroelectric crystal structuring. The statistical frequency doubling theory is developed for such structures. The effect of the number of random layers and variation in their thicknesses on the second harmonic conversion efficiency is clarified. It is demonstrated that a proper choice of the intermediate layer thickness can enhance or suppress the conversion efficiency. A new type of the Maker-fringes-like second harmonic intensity oscillations is predicted. (๑) 2017 Optical Society of America

OCIS codes: (190.2620) Harmonic generation and mixing; (190.4223) Nonlinear wave mixing.

http://dx.doi.org/10.1364/ao.XX.XXXXXX

At present, nonlinear photonic crystals (NPCs) are widely used for conversion of coherent laser radiation into new frequencies [1,2]. Nonlinear photonic crystals combine the high conversion efficiency, compactness, and exceptional universality in designing structures for target nonlinear optical processes. For these purposes, the electric field poling of ferroelectric crystals was developed [3]. At the same time, the local impact methods for structuring ferroelectrics have recently been proposed to fabricate NPCs. These methods include electron beam writing [4, 5], atomic force microscope writing [6, 7], and direct laser beam pattering $[8,9]$. The advantage of these methods is the possibility of fabricating arbitrary high-quality domain structures on the submicrometer and nanosized scales [10]. The drawback of these methods is the restriction imposed on the writing area size, which typically varies within $0.020 .2 \mathrm{~cm}$. Fabrication of relatively large (about $1 \mathrm{~cm}$ and more) structures faces certain difficulties. Although these structures can gradually be formed, the unavoidable random phase shifts at the transition between specific regular sections are caused by insufficiently accurate sample positioning during fabrication, which can result in the low nonlinear conversion efficiency.

The statistical phenomena related to the randomness of the nonlinear media parameters drew attention to nonlinear optics long time ago (see studies [11, 12] and monograph [13] and references therein). The random nonlinearity distribution leads to phase errors, which disturb phase matching [14]. On the other hand, it suggests the random quasi-phase matching, which enables the widely tunable $[15,16]$ and broadband frequency conversion [17]. The earlier studies addressed to either the continuously distributed random variations in domain thicknesses along the structures [18-22] or random fluctuations of the domain wall positions in regular structures [23].

In view of the aforesaid, the effect of random phase shifts on the nonlinear optical processes in NPCs fabricated by the new local impact methods is of special interest. In this work, we investigate the second harmonic generation (SHG) in an assembled nonlinear photonic crystal with periodical inserted random layers and establish a new type of the second harmonic (SH) intensity oscillations.

We consider the NPC design shown in Fig. 1. The NPC structure is assembled from periodically poled structures mediated by uniform layers with the random thickness fluctuations. The average random layer thickness was taken to be equal to a semiperiod of the periodic structure and the layer thickness fluctuations were assumed to obey the normal distribution law (with the standard deviation $\xi_{2 q}$ ). For certainty, we assume the number of random layers to be equal to the number $N+1$ of the periodically poled structures consisting of $2 M+1$ domains. In the undepleted fundamental wave approximation, the $\mathrm{SH}$ amplitude is governed by

$$
A_{2}=-i \beta A_{1}^{2} F(L)
$$

where $\beta=4 \pi k_{2} \chi^{(2)} / n_{2}$ is the nonlinear coupling coefficient, $A_{1}$ is the fundamental frequency amplitude, $k_{2}$ is the $\mathrm{SH}$ wavevector, $n_{2}$ is the $\mathrm{SH}$ refractive index, $\chi^{(2)}$ is the quadratic nonlinear susceptibility, and $L$ is the propagation coordinate. The function $F(L)$ describes the contribution of the structure to the process and takes the simplest form $F(L)=L$ under the phase matching condition.

According to Fig. 1, the SH amplitude is determined by the superposition of the amplitudes generated by the uniform $(u)$ 


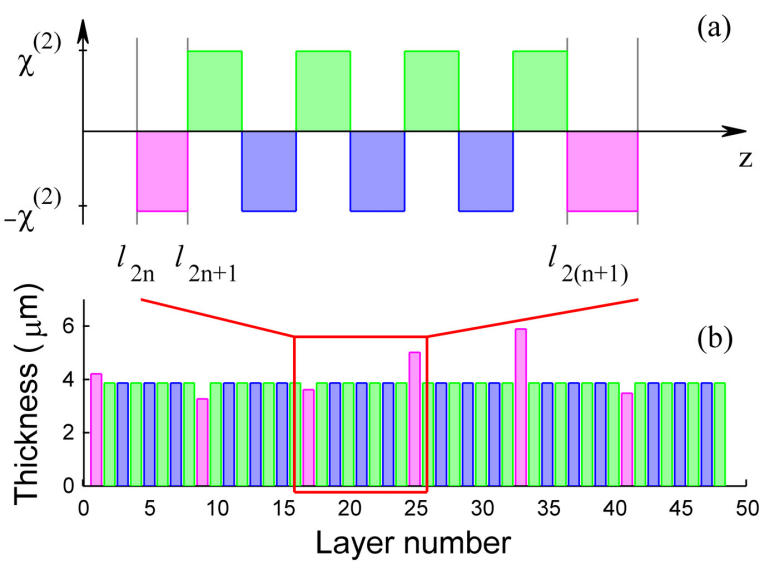

Fig. 1. (a) Nonlinearity modulation in the assembled structure composed of alternating periodic layers (blue and green) and random uniform layers (magenta). (b) Layer thicknesses vs layer numbers in the structure.

and periodically poled $(p)$ layers

$$
F(L)=\sum_{n=0}^{N}\left[F_{u}\left(2 n, 2 n+1 ; \delta_{2 n}\right)+F_{p}\left(2 n, 2(n+1) ; L_{p}\right)\right] .
$$

Here, the functions $F_{u}$ and $F_{p}$ correspond to the uniform and periodically poled structure parts, which are

$$
\begin{aligned}
& F_{u}\left(2 n, 2 n+1 ; \delta_{2 n}\right)=\frac{1}{i \Delta k}\left(e^{i \Delta k l_{2 n+1}}-e^{i \Delta k l_{2 n}}\right), \\
& F_{p}\left(2 n+1,2(n+1) ; L_{p}\right)=\int_{l_{2 n+1}}^{l_{2(n+1)}} g(z) e^{i \Delta k z} d z .
\end{aligned}
$$

In Eq. (3) and Eq. (4), $l_{n}$ is the layer coordinate $\left(l_{0}=0\right), \Delta k=$ $k_{2}-2 k_{1}$ is the wavevector mismatch, $g(z)$ is the alternating periodic function of the coordinate, $\delta_{2 n}=l_{2 n+1}-l_{2 n}$ is the uniform layer thickness (random variable), $L_{p}=l_{2(n+1)}-l_{2 n+1}=$ $\frac{1}{2}(2 M+1) \Lambda$ is the periodic part thickness, and $M$ is the number of periods. It can be shown that $l_{2 n}=\sum_{q=0}^{n-1} \delta_{2 q}+n L_{p}$.

Eq. (4) can be transformed to

$$
F_{p}\left(2 n+1,2(n+1) ; L_{p}\right)=-i \frac{2}{\pi} L_{p} e^{i \Delta k l_{2 n+1}},
$$

where $\Delta k l_{2 n+1}$ is the phase between the $\mathrm{SH}$ wave and polarization induced at the double frequency.

Taking into account Eq. (3) and Eq. (5), we arrive at

$$
F=-i \sum_{n=0}^{N}\left[\left(\frac{2 L_{p}}{\pi}+\frac{1}{\Delta k}\right) e^{i \Delta k l_{2 n+1}}-\frac{1}{\Delta k} e^{i \Delta k l_{2 n}}\right] .
$$

Further, with the accuracy $\mu=\pi /\left(2|\Delta k| L_{p}\right)=\Lambda / 4 L_{p} \ll 1$ (according to the parameters of the structure specified below $\mu \approx 10^{-2}$ ), we use Eq. (6) in the form

$$
F \approx-i \sum_{n=0}^{N}\left[\left(\frac{2 L_{p}}{\pi}\right) e^{i \Delta k l_{2 n+1}}\right] .
$$

As a result, the $\mathrm{SH}$ intensity is

$$
\begin{aligned}
I_{2}=\left|A_{2}(L)\right|^{2}=\beta^{2} I_{1}^{2}\left(\frac{2 L_{p}}{\pi}\right)^{2} & \\
& \times \sum_{n_{1}, n_{2}=0}^{N}\left[e^{i \Delta k\left(l_{2 n_{1}+1}-l_{2 n_{2}+1}\right)}\right],
\end{aligned}
$$

where

$$
\Delta k\left(l_{2 n_{1}+1}-l_{2 n_{2}+1}\right)=\Delta k\left[\left(n_{1}-n_{2}\right) L_{p}+\sum_{q=n_{2}}^{n_{1}} \delta_{2 q}\right] .
$$

Assume the uniform layer thickness to be

$$
\delta_{2 q}=\left(1+\sigma \xi_{2 q}\right) \Lambda / 2,
$$

where $\sigma \xi_{2 q}$ specifies the random fluctuations from the modulation semiperiod, $\sigma$ is the dispersion, $\xi_{2 q}$ is the random quantity $\left(\left\langle\xi_{2 q}\right\rangle=0,\left\langle\xi_{2 q}^{2}\right\rangle=1\right)$ with the fluctuations for different $q$ being statistically independent. Statistical averaging of Eq. (8), according to Eq. (10) and Eq. (9), yields

$$
\begin{aligned}
\left\langle I_{2}\right\rangle=\beta^{2} I_{1}^{2}\left(\frac{2}{\pi} L_{p}\right)^{2} & \\
& \times \sum_{n_{1}, n_{2}=0}^{N, N} \exp \left(-\frac{(\pi \sigma)^{2}}{2}\left|n_{1}-n_{2}\right|\right) .
\end{aligned}
$$

If $(\pi \sigma)^{2} \gg 1$, then the summation of the $\mathrm{SH}$ amplitudes will be incoherent and we obtain

$$
\left\langle I_{2}^{n c h}\right\rangle=\beta^{2} I_{1}^{2}\left(\frac{2}{\pi} L_{p}\right)^{2}(N+1) .
$$

At the coherent addition of the $\mathrm{SH}$ amplitudes $(\sigma=0)$, we have

$$
I_{2}^{c h}=\beta^{2} I_{1}^{2}\left(\frac{2}{\pi} L_{p}\right)^{2}(N+1)^{2} .
$$

After double summation in Eq. (11), we obtain the following general result:

$$
\begin{aligned}
\left\langle I_{2}\right\rangle=\beta^{2} & I_{1}^{2}\left(\frac{2}{\pi} L_{p}\right)^{2}\left(1-e^{-\gamma}\right)^{-2} \\
& \times\left[2(N+1)-2(N+2) e^{-\gamma}+2 e^{-\gamma(N+2)}\right. \\
& \left.-(N+1)\left(1-e^{-\gamma}\right)^{2}\right] .
\end{aligned}
$$

Here, $2 \gamma=(\pi \sigma)^{2}$ is the phase dispersion. In the limit $\gamma \rightarrow 0$ Eq. (14) is reduced to Eq. (13), while at $\gamma \gg 1$ we obtain Eq. (12). The results of single random implementations are intermediate between these two cases.

We consider frequency doubling of Nd:YAG laser radiation at a wavelength of $1.064 \mu \mathrm{m}$. The lithium niobate crystal was chosen as a nonlinear medium with the refractive index dispersion from [24]. The wavevector mismatch for the $e$ - ee interaction is $\Delta k \approx 0.92 \mu \mathrm{m}^{-1}$. Thus, the first-order quasi-phasematched SHG requires modulation on the nonlinearity with a period of $\Lambda=6.78 \mu \mathrm{m}$.

A set of random uniform layer thicknesses is simulated using Eq. (10). In practice, it is reasonable to take into account the absolute accuracy of sample positioning $\sigma \Lambda / 2$ instead of the relative $\sigma$ value. As an actual positioning accuracy, we can 
choose a value of $1 \mu \mathrm{m}$ corresponding to the relative standard deviation $\sigma \approx 0.15$.

Eq. (6) is used to calculate the SH intensity on the coordinate $l_{n}$ for a series of random implementations of the structure. The calculated data are shown in Fig. 2. The parameters used were $\sigma=0.15, M=30, N=24$, and a crystal thickness of $L \approx 5$ $\mathrm{mm}$. One can see that the monotonic $\mathrm{SH}$ intensity growth in the periodically poled layers can be changed for a decrease due to the random phase shifts acquired in the random uniform layers and vice versa.

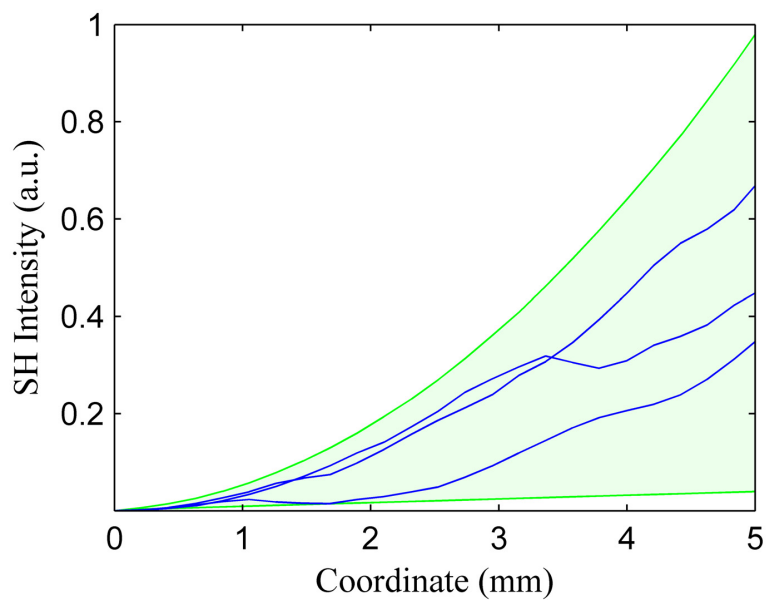

Fig. 2. Dependences of the SH intensity on the coordinate (blue) for a series of random implementations of the structure calculated using Eq. (6) $(\sigma=0.15, M=30, N=24$, and $L \approx 5$ $\mathrm{mm})$. The outlined region is constrained by the results calculated using Eq. (12) (lower curve) and Eq. (13) (upper curve).

Fig. 3 shows the $\mathrm{SH}$ intensity as a function of the standard layer thickness deviation for ten random implementations calculated using Eq. (6). The data averaging reveals the nonmonotonic behavior, which is caused by a small number of implementations. One can see that the average $\mathrm{SH}$ intensity described by Eq. (14) derived using the developed statistical theory fits well the results of the random implementations.

We consider the process in the extreme cases when the uniform layer thickness is exactly equal to the regular structure semiperiod or period. The first case corresponding to the perfect quasi-phase matching is mathematically expressed as $\delta_{n}=$ $\Lambda / 2$ and $\sigma=0$. In this case, the $\mathrm{SH}$ intensity calculated using Eq. (12) obeys the quadratic law corresponding to the quasiphase-matched SHG (Fig. 2).

An interesting situation is observed when the uniform layer thickness is exactly equal to the regular structure period $\left(\delta_{n}=\right.$ $\Lambda$ and $\sigma=0$ ). Basically, the SHG phase mismatch leads to the $\mathrm{SH}$ intensity oscillations along the media. In the case under study, a new type of the $\mathrm{SH}$ intensity oscillations along the structure is numerically predicted, as can be seen in Fig. 4 . The oscillation period is $\Lambda_{N P C}=(2 M+3) \Lambda$, which differs from the $\mathrm{SH}$ intensity oscillations with period $\Lambda$ in uniform media. The nature of these oscillations is as follows. The phase mismatch of interacting waves accumulated in the middle of the uniform layer attains its maximum and the destructive interference of $\mathrm{SH}$ amplitudes takes place. The inverse phenomenon (i.e., constructive interference) occurs in the next uniform layer. The oscillations are very similar to those corresponding to the

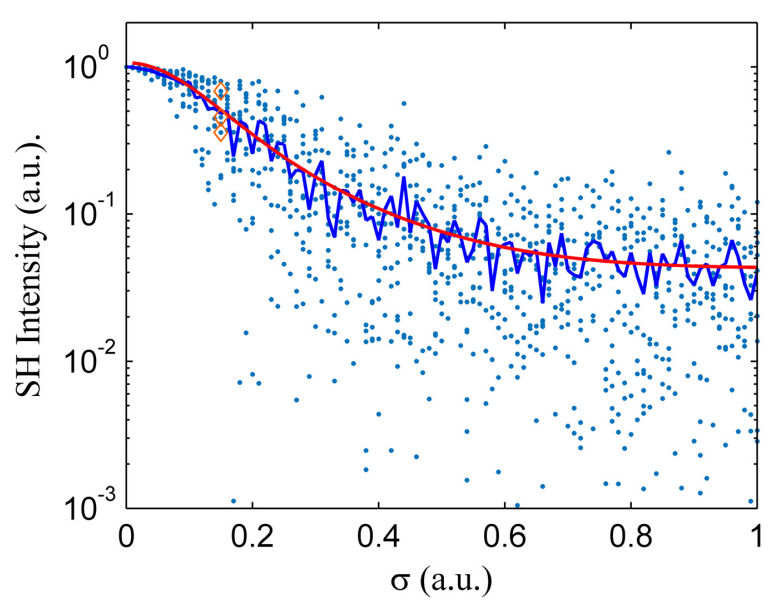

Fig. 3. Dependence of the SH intensity on the standard random layer deviation for ten random implementations (blue points), their average values (blue curve), and average intensity calculated using Eq. (14)(red curve).

Maker fringes observed in uniform media at the phase mismatch (see, for example, [22]). The oscillation amplitude can be enhanced by a factor of $4(M+1)^{2}$. Note that the similar results can be obtained by excluding the intermediate layers from the structure $\left(\delta_{n}=0\right.$ and $\left.\sigma=0\right)$. In addition, this behavior will result in the $\mathrm{SH}$ intensity oscillations in the spectral and angular dependences shown in Fig. 5(a). They can be treated as quasi-phase-matched Maker fringes. For this purpose, the fundamental wavelength was tuning and thicknesses of all the layers were divided by $\cos \theta$, where angle $\theta$ is counted from the quasi-phase-matching direction. There are two spectral peaks that exhibit a long-wavelength shift upon rotation of the structure. The spectral spacing between the peaks grows with a decrease in $M$. Fig. 5(b) shows the corresponding angular dependence of the $\mathrm{SH}$ spectral intensity for a periodically poled crystal $\left(\delta_{n}=\Lambda / 2\right)$.

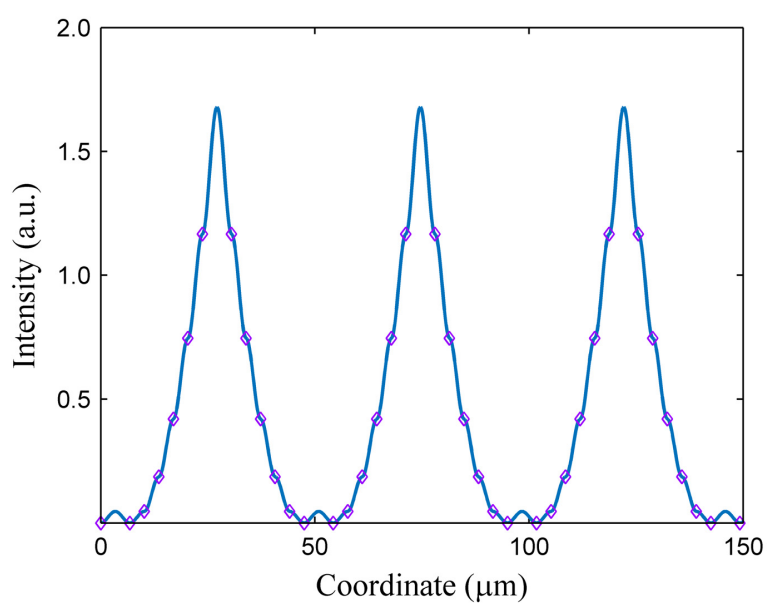

Fig. 4. SH intensity oscillations in the structure calculated numerically (solid line) and using Eq. (6) (points) at the parameters of $\delta_{n}=\Lambda, \sigma=0$ and $M=2$.

Thus, we have developed the statistical theory of frequency 
(a)

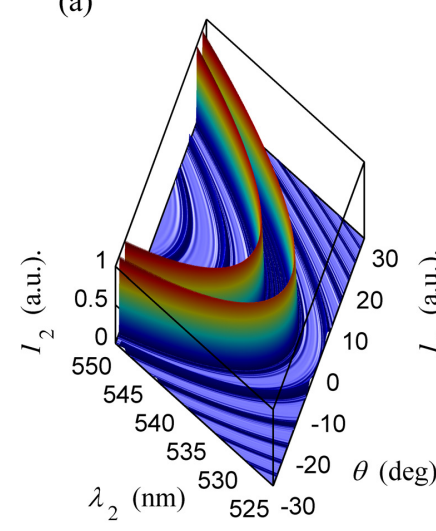

(b)

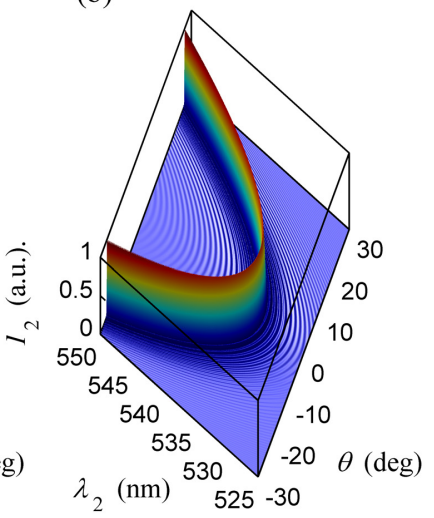

Fig. 5. Normalized SH spectral intensity vs NPC rotation angle $(M=30$ and $N=7)$ : (a) $\delta_{n}=\Lambda$ and (b) $\delta_{n}=\Lambda / 2$.

doubling in assembled nonlinear photonic crystals with the periodic structures mediated by the layers of random thickness. Our analysis shows that the $\mathrm{SH}$ intensity in the structure under study in the limit incoherent case is $I_{2} \propto N M^{2}$ instead of $I_{2} \propto(N M)^{2}$ corresponding to the regular structure (coherent summation of the contribution of layers). It means that the structure under study is inferior to the regular one in $N$ times. It was shown that the second harmonic oscillations enhanced by the quasi-phase matching take place in the absence of periodic compensations of the phase mismatch.

\section{FUNDING}

Council of the President of the Russian Federation (MK2908.2015.2); Russian Foundation for Basic Research (RFBR) (1502-03838); The authors thank I.S. Baturin for fruitful discussions and D. Agapov for help.

\section{REFERENCES}

1. A. Arie, N. Voloch, Periodic, quasi-periodic, and random quadratic nonlinear photonic crystals, Laser Photon. Rev. 4, 355 (2010).

2. V. Pasiskevicius, G. Strömqvist, F. Laurell, C. Canalias, Quasi-phase matched nonlinear media: Progress towards nonlinear optical engineering, Opt. Mat. 34, 513 (2012).

3. M. Yamada, N. Nada, M. Saitoh, K. Watanabe, First-order quasi-phase matched $\mathrm{LiNbO}_{3}$ waveguide periodically poled by applying an external field for efficient blue second-harmonic generation, Appl. Phys. Lett. 62 , 435 (1993).

4. V. Ya. Shur, D. S. Chezganov, A. R. Akhmatkhanov, D. K. Kuznetsov, Domain patterning by electron beam of $\mathrm{MgO}$ doped lithium niobate covered by resist, Appl. Phys. Lett. 106, 232902 (2015).

5. J. L. Hart, S. Liu, A. C. Lang, A. Hubert, A. Zukauskas, C. Canalias, R. Beanland, A. M. Rappe, M. Arredondo, M. L. Taheri, Electron-beaminduced ferroelectric domain behavior in the transmission electron microscope: Toward deterministic domain patterning, Phys. Rev. B 94, 174104 (2016).

6. L. V. Simagina, E. D. Mishina, S. V. Semin, N. A. llyin, T. R. Volk, R. V. Gainutdinov, L. I. Ivleva, Second harmonic generation in microdomain gratings fabricated in strontium-barium niobate crystals with an atomic force microscope, J. Appl. Phys. 110, 052015 (2011).

7. T. R. Volk, R. V. Gainutdinov, Ya. V. Bodnarchuk, L. I. Ivleva, Creation of domains and domain patterns on the nonpolar surface of
$\mathrm{Sr}_{x} B a_{1-x} N b_{2} \mathrm{O}_{6}$ crystals by atomic force microscopy, JETP Lett. $\mathbf{9 7}$, 483 (2013).

8. S. Kroesen, K. Tekce, J. Imbrock, C. Denz, Monolithic fabrication of quasi phase-matched waveguides by femtosecond laser structuring the $\chi^{2}$ nonlinearity, Appl. Phys. Lett. 107, 101109 (2015).

9. X. Chen, P. Karpinski, V. Shvedov, K. Koynov, B. Wang, J. Trull, C. Cojocaru, W. Krolikowski, Y. Sheng, Ferroelectric domain engineering by focused infrared femtosecond pulses, Appl. Phys. Lett. 107, 141102 (2015).

10. V. Ya. Shur, A. R. Akhmatkhanov, I. S. Baturin, Micro- and nanodomain engineering in lithium niobate, Appl. Phys. Rev. 2, 040604 (2015).

11. I. Freund, Critical Harmonic Scattering in $\mathrm{NH} 4 \mathrm{Cl}$, Phys. Rev. Lett. 19, 1288 (1967).

12. I. Freund, Harmonic scattering and critical point correlations in $\mathrm{NH} 4 \mathrm{Cl}$, Chem. Phys. Lett. 1, 551 (1968).

13. S. A. Akhmanov, A. S. Chirkin, Statistical Phenomena in Nonlinear Optics, MSU Publishing, 1971 (in Russian).

14. M. M. Fejer, G. A. Magel, D. H. Jundt, R. L. Byer, Quasi-phasematched second harmonic generation: tuning and tolerances, IEEE J. Quantum Electron. 28, 2631 (1992).

15. A. S. Aleksandrovsky, A. M. Vyunishev, A. I. Zaitsev, V. V. Slabko, Random quasi-phase-matched conversion of broadband radiation in a nonlinear photonic crystal, Phys. Rev. A 82, 055806 (2010).

16. A. S. Aleksandrovsky, A. M. Vyunishev, A. I. Zaitsev, G. I. Pospelov, V. V. Slabko, Diagnostics of fs pulses by noncollinear random quasiphase-matched frequency doubling, Appl. Phys. Lett. 99, 211105 (2011).

17. A. S. Aleksandrovsky, A. M. Vyunishev, A. I. Zaitsev, V. V. Slabko, Random quasi-phase-matched nonlinear optical conversion of supercontinuum to the ultraviolet, Appl. Phys. Lett. 103, 251104 (2013).

18. E. A. Vinogradov, A. N. Vtyurin, A. F. Goncharov, G. N. Zhilin, I. S. Kabanov, V. F. Shabanov, Generation of the 2nd optical harmonic in a crystal with macroscopic inhomogeneities, Opt. Spektr. 52, 159 (1982) (in Russian).

19. E. Yu. Morozov, A. A. Kaminskii, A. S. Chirkin, D. B. Yusupov, Second optical harmonic generation in nonlinear crystals with a disordered domain structure, JETP Lett. 73, 647 (2001).

20. E. Yu. Morozov, A. S. Chirkin, Stochastic quasi-phase matching in nonlinear-optical crystals with an irregular domain structure, Quant. Electron. 34, 227 (2004).

21. M. Baudrier-Raybaut, R. Haïdar, P. Kupecek, P. Lemasson, E. Rosencher, Random quasi-phase-matching in bulk polycrystalline isotropic nonlinear materials, Nature 432, 374 (2004).

22. A. S. Aleksandrovsky, A. M. Vyunishev, I. E. Shakhura, A. I. Zaitsev, A. V. Zamkov, Random quasi-phase-matching in a nonlinear photonic crystal structure of strontium tetraborate, Phys. Rev. A 78, 031802 (2008).

23. I. V. Shutov, A. S. Chirkin, Simulation of the random violation of the quasi-phase-matching condition in optical parametric process, Quant. Electron. 39, 691 (2009).

24. D. H. Jundt, Temperature-dependent Sellmeier equation for the index of refraction, $n_{e}$, in congruent lithium niobate, Opt. Lett. 22, 1553 (1997). 